\title{
Updates of Pulmonary Embolism
}

\author{
Mohamed H. A. Elsayed
}

\section{Background}

Pulmonary embolism (PE) is the third most common cause of cardiovascular death worldwide, behind myocardial infarction and stroke. Due to pulmonary bed obstruction, PE can result in acute right ventricular (RV) failure which is a lifethreatening condition. Because most patients ultimately die within the first hours of presentation, early diagnosis is very important (1). Mortality due to pulmonary embolism varies greatly, depending on various factors including age, comorbid conditions, and stability on presentation. Patients with low-risk PE have a 1-year survival rate over 95\%. In contrast, patients presenting with high risk PE and hemodynamic instability have an approximately $40 \%$ mortality rate within 90 -days. In this review, we will discuss the basic pathophysiology of PE, risk factors for developing PE, and standard diagnostic testing modalities. We will also cover risk stratification of patients presenting with $\mathrm{PE}$ and the implications for treatment and disposition. While no exact epidemiological data are available, the incidence of $\mathrm{PE}$ is estimated to be approximately 60 to 70 per 100,000, and that of venous thrombosis approximately 124 per 100,000 of the general population (2). The European guidelines for the diagnosis and management of PE report annual incidence rates of venous thrombosis and PE of approximately 0.5 to 1.0 per 1000 inhabitants (3). However, the actual figures are likely to be substantially higher because silent PE can develop in up to $40 \%$ to $50 \%$ of patients with deep vein thrombosis (DVT) (3).

\section{Pathophysiology}

PE occurs when clots formed in the deep venous system dislodge or break loose, travel through the heart, and become lodged in the pulmonary vasculature. While small PE can frequently lyse spontaneously, larger PE can cause a sudden and persistent rise in pulmonary artery pressure, which can lead to circulatory collapse. The effects of an embolus depend on the extent to which it obstructs the pulmonary circulation, the duration over which that obstruction accumulates, and the pre-existing state of the patient, which has been defined only imprecisely. Some humoral mediators (for example, serotonin or thromboxane from activated platelets) can probably produce vasospasm in non-embolised segments of the lung. As a result, a degree of pulmonary hypertension may develop disproportionate to the amount of vasculature that is mechanically occluded (4). The first which is most common presentation is dyspnea with or without pleuritic pain and hemoptysis (acute minor pulmonary embolism). The second presentation is hemodynamic instability. It is associated with acute massive pulmonary embolism. The third and least common presentation mimics heart failure or pneumonia, especially in the elderly (subacute massive pulmonary embolism)

\section{Risk stratification}

Risk stratification play an important role allowing physicians to deliver the optimal treatment to the patients. PE has been shown to cover a wide spectrum of clinical severity with early mortality rates ranging between less than $1 \%$ and above $50 \%$ (5). A substantial body of evidence suggests that advanced risk stratification can distinguish between intermediate and low clinical risk in the normotensive patients. Advanced age, major underlying conditions (cancer and cardiac or respiratory 
disease), clinical signs of right ventricular dysfunction (tachycardia and hypotension) and hypoxemia are the main clinical determinants of the outcome of patients with PE (6) . There are multiple factors that can increase one's chance of developing DVT and PE. These factors can be inherited, acquired over time, or provoked. Various inherited conditions increase a patient's risk for venous thromboembolism (VTE), the most common of which are factor V Leiden and prothrombin gene mutation (G20210A) with population prevalences of approximately $4 \%$ to $5 \%$ and $2 \%$ to $4 \%$, respectively(7). In patients with factor V Leiden, the increased risk of VTE is 2- to 7fold in heterozygous individuals and up to 40-fold in those who are homozygous (8). The most common acquired risk factors for VTE include: increasing age, venous insufficiency, obesity, smoking, rheumatologic conditions, cardiovascular disease, previous VTE, and antiphospholipid antibody syndrome. The mechanism behind increasing age appears to be that naturally circulating anticoagulants (protein $\mathrm{C}$ and protein $\mathrm{S}$ ) decrease more than pro-coagulation factors over time, creating an increased prothrombotic state. This, combined with increased venous stasis in the lower extremities, increases the rates of DVT and PE in the elderly population. Obesity is a known risk factor for VTE, and data from the Nurses' Health Study found that, among the most obese subjects (body mass index $>35$ ), there was a 6-fold increase in risk when compared to normal-weight subjects (9). There are variable classifications used to define risks from PE, we will categorize patients as low-risk, intermediate-risk, and high-risk(10).

\section{High risk}

Patients with PE who present with hypotension, syncope, bradycardia, or the inability to maintain adequate oxygenation are at risk for sudden death, even with appropriate treatment. Patients with large PE and residual clot present in the heart ("clot-intransit") or iliofemoral veins should also be considered at high risk for decompensation. High-risk patients often require emergent intervention and admission to the intensive care unit.

\section{Intermediate risk}

Patients who presents with end organ damage but are hemodynamically stable can be considered intermediate risk. Right heart strain on echocardiography, in the setting of a large PE, is also associated with an increased risk of clinical deterioration and/or short-term death. Other factors, such as an elevated troponin indicating cardiac ischemia, altered mental status, and the presence of co-morbid illness have also been correlated with early clinical deterioration and higher incidence of short-term death (10).

\section{Low risk}

Patients found to have PE without evidence of end organ damage or hemodynamic instability may be able to be safely discharged early after initiation of anticoagulation. In an analysis of 298 consecutive patients with PE, we found that $2 / 3$ of patients suffered no clinical deterioration and required no hospital-based interventions after PE. This suggests that a large proportion of patients derive no benefit from hospitalization after PE, and may be safe for discharge from the ED. hypotension, hypoxia, history of coronary artery disease, evidence of right heart strain, and residual DVT were all found to be independently associated with clinical deterioration. Patients who present with none of these risk factors are likely at low risk for early clinical deterioration and are ideal candidates for early discharge after initiation of anticoagulation(11). 


\section{Clinical presentation:}

The effects of an embolus depend on the extent to which it obstructs the pulmonary circulation, the duration over which that obstruction accumulates, and the pre-existing state of the patient, which has been defined only imprecisely. In general, a patient who has pre-existing cardiopulmonary disease or who is old, frail or debilitated will be more sensitive to the effects of pulmonary embolism than a patient who was well until the embolic event occurred. Disregarding chronic thromboembolic pulmonary hypertension, it is convenient to classify pulmonary embolism into three main types. The first and most common presentation is dyspnoea with or without pleuritic pain and haemoptysis (acute minor pulmonary embolism). The second presentation is haemodynamic instability, which is associated with acute massive pulmonary embolism. The third and least common presentation mimics heart failure or indolent pneumonia, especially in the elderly (subacute massive pulmonary embolism)(4)

Acute minor pulmonary embolism

Acute small PE has a good prognosis, with a three-month mortality rate $<1 \%$. It can be asymptomatic or accompanied only by tachypnea ( $\geq 24$ breaths/min) and tachycardia ( $\geq 100$ beats/min), or it can manifest itself as only a mild elevation of body temperature. Silent PE is not a rare occurrence; a study published by Meignan et al in 2000 involving 622 patients with proximal DVT reported silent PE diagnosed by routine lung perfusion scans in $40 \%$ to $50 \%$ of the patients.

Acute massive pulmonary embolism

The most severe form of PE is an acute massive PE (ie, high risk) with mortality rates exceeding 20\% irrespective of treatment. Acute massive PE is characterized by hemodynamic instability (ie, manifesting itself as syncope, persistent hypotension and cardiogenic shock (with hypotension defined as a sudden fall in systolic blood pressure to $<90 \mathrm{mmHg}$ or more, or by $\geq 40 \mathrm{mmHg}$ from baseline). This may occur so rapidly that syncope is either the presenting feature or easily induced by a relatively minor cardiovascular stress. If the degree of obstruction is sufficient, death occurs almost immediately. The fall in aortic pressure and the rise in right ventricular pressure may cause ischaemia of the right ventricle through a critical reduction of right coronary perfusion. Electromechanical dissociation is the most frequent cause of final cardiac arrest.

\section{Subacute massive pulmonary embolism}

This is caused by multiple small or moderately sized emboli that accumulate over several weeks. The rises in the right ventricular end diastolic and right atrial pressures are of a lesser extent than in acute massive pulmonary embolism since there is time for adaptation to occur and the degree of right ventricular failure is less for a given degree of pulmonary artery obstruction. The main symptoms are increasing dyspnoea and decreasing exercise tolerance. There is often an associated dry cough. The breathlessness is usually out of proportion to all other findings, and there may be central cyanosis

\section{Diagnostic procedures}

The clinical diagnosis of pulmonary embolism is difficult, particularly when there is coexisting heart or lung disease. Nearly all patients with pulmonary embolism will have one or more of the following clinical features; dyspnoea of sudden onset, tachypnoea (>20 breaths/minute), or chest pain (pleuritic or substernal); if the 
clinician remembers these three features, the possibility of pulmonary embolism will rarely be overlooked. When these clinical features are associated with ECG signs of right ventricular strain and/or radiologic signs of plump hilum, pulmonary infarction or oligaemia, the likelihood of pulmonary embolism is high, and it is further strengthened in the presence of risk factors for venous thromboembolism. On the contrary, the absence of all these three clinical features virtually excludes the diagnosis of pulmonary embolism.

\section{Electrocardiography}

The most common ECG abnormalities in the setting of pulmonary embolism are tachycardia and nonspecific ST-T wave abnormalities. The finding of $\mathrm{S}_{1} \mathrm{Q}_{3} \mathrm{~T}_{3}$ is nonspecific and insensitive in the absence of clinical suspicion for pulmonary embolism. The classic findings of right heart strain and acute Cor-pulmonale are tall, peaked $\mathrm{P}$ waves in lead II ( $\mathrm{P}$ pulmonale); right axis deviation; right bundle-branch block; an $\mathrm{S}_{1} \mathrm{Q}_{3} \mathrm{~T}_{3}$ pattern; or atrial fibrillation. Unfortunately, only $20 \%$ of patients with proven pulmonary embolism have any of these classic electrocardiographic abnormalities.

\section{Chest radiography}

Chest radiographs are abnormal in most cases of pulmonary embolism, but the findings are nonspecific. Common radiographic abnormalities include atelectasis, pleural effusion, parenchymal opacities, and elevation of a hemidiaphragm. The classic radiographic findings of pulmonary infarction include a wedge-shaped, pleurabased triangular opacity with an apex pointing toward the hilus (Hampton hump) or decreased vascularity (Westermark sign). These findings are suggestive of pulmonary embolism but are infrequently observed. The abrupt tapering or cutoff of a pulmonary artery secondary to embolus (knuckle sign), cardiomegaly (especially on the right side of the heart), and pulmonary edema are other findings

\section{Echocardiography}

Acute PE may lead to RV pressure overload and dysfunction, which can be detected by echocardiography. Several echocardiographic findings have been proposed for noninvasive diagnosis of right ventricular dysfunction at the bedside, including right ventricular enlargement and/or hypokinesis of the free wall, leftward septal shift, and evidence of pulmonary hypertension.

\section{Blood tests}

In patients with a low or moderate suspicion of PE, a normal D-dimer level is enough to exclude the possibility of thrombotic PE, with a three-month risk of thromboembolic events being $0.14 \%$. D-dimer is highly sensitive but not specific (specificity around 50\%). In other words, a positive D-dimer is not synonymous with PE, but a negative D-dimer is, with a good degree of certainty, an indication of absence of a PE (12) The typical cut off is $500 \mu \mathrm{g} / \mathrm{L}$, although this varies based on the assay. As for arterial blood gas, it is characteristically reveals hypoxemia, hypocapnia, and respiratory alkalosis.

\section{Lung scintigraphy}

Ventilation-perfusion scintigraphy (V/Q scan) is an established diagnostic test for suspected PE. It is safe although a few allergic reactions have been described. The test is based on the intravenous injection of technetium (Tc)-99m-labelled macroaggregated albumin particles, which block a small fraction of the pulmonary capillaries and thereby enable scintigraphic assessment of lung perfusion. Perfusion scans are combined with ventilation studies, for which multiple tracers such as xenon- 
133 gas, Tc-99m-labelled aerosols, or Tc-99m-labelled carbon microparticles (Technegas) can be used. The purpose of the ventilation scan is to increase specificity: in acute PE, ventilation is expected to be normal in hypoperfused segments (mismatch)

\section{Computed tomography (CT, spiral or electron beam CT)}

Computed tomographic angiography has become the method of choice for imaging the pulmonary vasculature in patients with suspected PE. It allows adequate visualization of the pulmonary arteries down to at least the segmental level. In patients with a low or intermediate clinical probability of PE, a negative CT had a high negative predictive value for PE (96\% and $89 \%$, respectively), whereas this was only $60 \%$ in those with a high pre-test probability. Conversely, the positive predictive value of a positive CT was high (92-96\%) in patients with an intermediate or high clinical probability but much lower $(58 \%)$ in patients with a low pre-test likelihood of PE. Therefore, clinicians should be particularly cautious in case of discordancy between clinical judgement and the MDCT result.

\section{Magnetic resonance imaging}

With magnetic resonance imaging (MRI), evidence of pulmonary emboli may be detected by using standard or gated spin-echo techniques. Pulmonary emboli demonstrate increased signal intensity within the pulmonary artery. By obtaining a sequence of images, this signal that is originating from slow blood flow may be distinguished from pulmonary embolism. However, this remains a problem in pulmonary hypertension. MRI has a sensitivity of $85 \%$ and specificity of $96 \%$ for central, lobar, and segmental emboli but is inadequate for the diagnosis of subsegmental emboli.

\section{Pulmonary angiography}

Pulmonary angiography is the historical criterion standard for the diagnosis of pulmonary embolism. Positive results consist of a filling defect or sharp cutoff of the affected artery

\section{Treatment}

\section{Hemodynamic management}

Acute RV failure with resulting low systemic output is the leading cause of death in patients with high-risk PE. Therefore, supportive treatment is vital in patients with PE and RV failure. Experimental studies indicate that aggressive volume expansion is of no benefit and may even worsen RV function by causing mechanical overstretch, or by reflex mechanisms that depress contractility(13). On the other hand, modest (500 $\mathrm{mL}$ ) fluid challenge may help to increase cardiac index in patients with PE, low cardiac index, and normal $\mathrm{BP}(14)$

\section{The role of thrombolytic therapy}

Fibrinolytic agents have been tested in randomized trials over almost one-half a century, and those currently approved for clinical use were recently reviewed (15). A meta-analysis of 15 trials involving a total of 2,057 patients showed that fibrinolysis reduced overall mortality (odds ratio [OR]: 0.59 ; $95 \% \mathrm{CI}: 0.36$ to 0.96 ) and achieved a significant reduction in the combined endpoint of death or treatment escalation (OR: 0.34 ; 95\% CI: 0.22 to 0.53 ), PE-related mortality (OR: 0.29 ; 95\% CI: 0.14 to 0.60 ), and PE recurrence (OR: $0.50 ; 95 \%$ CI: 0.27 to 0.94 ). At the same time, major hemorrhage (OR: 2.91; 95\% CI: 1.95 to 4.36) and fatal or intracranial bleeding 
(OR: 3.18 ; 95\% CI: 1.25 to 8.11 ) were significantly more frequent among patients receiving thrombolysis. Accelerated regimens administered over 2 hours are preferable to prolonged infusions of first-generation thrombolytic agents over 12-24 hours. Reteplase and desmoteplase have been tested against recombinant tissue plasminogen activator (rtPA) in acute PE, with similar results in terms of haemodynamic parameters; tenecteplase was tested against placebo in patients with intermediate-risk PE.

\section{Oral anticoagulants}

These drugs prevent new clots from forming while the body works to break up the clots. Heparin is a frequently used anticoagulant that can be given through the vein or injected under the skin. Most people diagnosed with pulmonary embolism will need injections of heparin for at least five days, then usually continue to take warfarin only. A newer class of anticoagulants, referred to as novel oral anticoagulants (NOACs) like dabigatran, rivaroxaban and apixaban, has been tested and approved for treatment of venous thromboembolism, including pulmonary embolism. Like any medication, anticoagulants may have side effects, and effects will vary from person to person. One of the most important problems is bleeding more easily and excessively. Because of this, you will need regular blood tests to make sure you're on the best dose

\section{What about vena cava filter?}

A catheter can be used to position a filter in the inferior vena cava. This filter can help keep clots from being carried into your lungs. This procedure is typically reserved for people who can't take anticoagulant drugs or when anticoagulant drugs don't work well enough or fast enough. Some filters can be removed when they are no longer needed.

\section{Percutaneous Mechanical Thrombectomy (PMT)}

Several percutaneous approaches have been used alone or in combination in patients with an absolute contraindication to thrombolysis. These include: 1) thrombus fragmentation with a rotating pigtail catheter; 2) aspiration thrombectomy; 3) rheolytic thrombectomy; and 4) suction embolectomy. Thrombus fragmentation techniques using balloon angioplasty or rotation of pigtail catheters are probably the earliest examples of catheter-based intervention for acute PE. The optimal PMT strategy to use in patients with an absolute contraindication to systemic fibrinolysis is best determined on an individualized basis.

\section{Surgical embolectomy}

The first successful surgical pulmonary embolectomy was performed in 1924, several decades before the introduction of medical treatment for PE. Pulmonary embolectomy is technically a relatively simple operation. The site of surgical care does not appear to have a significant effect on operative outcomes, and thus patients need not be transferred to a specialized cardiothoracic Centre if on-site embolectomy using extracorporeal circulation is possible. Transportable extracorporeal assistance systems with percutaneous femoral cannulation can be helpful in critical situations, ensuring circulation and oxygenation until definitive diagnosis

\section{Conclusion}

Recently published randomized trials and major cohort studies were able to clarify several important aspects related to the management of acute PE. In particular, age-adjusted D-dimer cutoff levels were helpful in optimizing the use of imaging procedures in patients with low pre-test clinical probability for the disease; the risks of full-dose systemic fibrinolysis, 
administered as primary treatment, were shown to outweigh its benefits in patients with intermediate-risk $\mathrm{PE}$; catheter-directed pharmacomechanical techniques emerged as a promising option for patients with indications to reperfusion treatment and a high bleeding risk; 4 new oral anticoagulant agents were approved for treatment and secondary prevention of VTE, showing noninferior efficacy and probably superior safety compared with traditional heparin/VKA regimens; retrievable vena cava filters were not found to improve 3-month or 6-month prognoses when inserted on top of anticoagulation treatment; and further evidence was provided in favor of extended or even indefinite anticoagulation for secondary prophylaxis after unprovoked PE.

\section{References}

1. Bělohlávek J, Dytrych V, Linhart A. Pulmonary embolism, part I: Epidemiology, risk factors and risk stratification, pathophysiology, clinical presentation, diagnosis and nonthrombotic pulmonary embolism. Experimental \& Clinical Cardiology. 2013;18(2):129-38.

2. Oger E. Incidence of venous thromboembolism: a community-based study in Western France. EPI-GETBP Study Group. Groupe d'Etude de la Thrombose de Bretagne Occidentale. Thrombosis and haemostasis. 2000;83(5):657-60.

3. Torbicki A, Perrier A, Konstantinides S, Agnelli G, Galie N, Pruszczyk P, et al. Guidelines on the diagnosis and management of acute pulmonary embolism: the Task Force for the Diagnosis and Management of Acute Pulmonary Embolism of the European Society of Cardiology (ESC). Eur Heart J. 2008;29(18):2276-315.

4. Riedel M. Acute pulmonary embolism 1: pathophysiology, clinical presentation, and diagnosis. Heart. 2001;85(2):229-40.

5. Meyer G, Vieillard-Baron A, Planquette B. Recent advances in the management of pulmonary embolism: focus on the critically ill patients. Annals of Intensive Care. 2016;6:19.

6. Aujesky D, Roy PM, Le Manach CP, Verschuren F, Meyer G, Obrosky DS, et al. Validation of a model to predict adverse outcomes in patients with pulmonary embolism. Eur Heart J. 2006;27(4):476-81.

7. Poort SR, Rosendaal FR, Reitsma PH, Bertina RM. A common genetic variation in the 3'-untranslated region of the prothrombin gene is associated with elevated plasma prothrombin levels and an increase in venous thrombosis. Blood. 1996;88(10):3698703.

8. Ginsburg KS, Liang MH, Newcomer L, Goldhaber SZ, Schur PH, Hennekens $\mathrm{CH}$, et al. Anticardiolipin antibodies and the risk for ischemic stroke and venous thrombosis. Annals of internal medicine. 1992;117(12):997-1002.

9. Goldhaber SZ, Grodstein F, Stampfer MJ, Manson JE, Colditz GA, Speizer FE, et al. A prospective study of risk factors for pulmonary embolism in women. Jama. 1997;277(8):642-5.

10. Sanchez O, Trinquart L, Caille V, Couturaud F, Pacouret G, Meneveau $\mathrm{N}$, et al. Prognostic factors for pulmonary embolism: the prep study, a prospective multicenter cohort study. American journal of respiratory and critical care medicine. 2010;181(2):168-73.

11.Kabrhel C, Okechukwu I. Factors associated with clinical deterioration shortly after PE. 2014;69(9):835-42.

12. Carrier M, Righini M, Djurabi RK, Huisman MV, Perrier A, Wells PS, et al. VIDAS D-dimer in combination with clinical pre-test probability to rule out pulmonary embolism. A systematic review of management outcome 
studies. Thrombosis and haemostasis. 2009;101(5):886-92.

13. Ghignone M, Girling L, Prewitt RM. Volume expansion versus norepinephrine in treatment of a low cardiac output complicating an acute increase in right ventricular afterload in dogs. Anesthesiology. 1984;60(2):132-5.

14. Mercat A, Diehl JL, Meyer G, Teboul JL, Sors H. Hemodynamic effects of fluid loading in acute massive pulmonary embolism. Critical care medicine. 1999;27(3):540-4.

15. Konstantinides S, Torbicki A, Agnelli G, Danchin N, Fitzmaurice D, Galie N, et al. Downloaded from Schindler TH, Svitil P, Vonk Noordegraaf A, Zamorano JL, Zompatori M. 2014 ESC guidelines on the diagnosis and management of acute pulmonary embolism. Eur Heart J. 2014;35:303369. 